\title{
Bulk Spin Polarization of Magnetite from Spin-Resolved Hard X-Ray Photoelectron Spectroscopy
}

\author{
M. Schmitt, ${ }^{1}$ O. Kirilmaz, ${ }^{1}$ S. Chernov ${ }^{2}$ S. Babenkov, ${ }^{2}$ D. Vasilyev, ${ }^{2}$ O. Fedchenko, ${ }^{2}$ \\ K. Medjanik, ${ }^{2}$ Yu. Matveyev, ${ }^{3}$ A. Gloskovskii, ${ }^{3}$ C. Schlueter, ${ }^{3}$ A. Winkelmann, ${ }^{4}$ \\ L. Dudy ${ }^{5}$ H.-J. Elmers, ${ }^{2}$ G. Schönhense, ${ }^{2}$ M. Sing, ${ }^{1}$ and R. Claessen ${ }^{1}$ \\ ${ }^{1}$ Physikalisches Institut and Würzburg-Dresden Cluster of Excellence ct.qmat, \\ Julius-Maximilians-Universität, 97074 Würzburg, Germany \\ ${ }^{2}$ Institut für Physik, Johannes Gutenberg-Universität, 55128 Mainz, Germany \\ ${ }^{3}$ DESY Photon Science, 22607 Hamburg, Germany \\ ${ }^{4}$ Academic Centre for Materials and Nanotechnology (ACMiN), \\ AGH University of Science and Technology, 30-059 Kraków, Poland \\ ${ }^{5}$ SOLEIL Synchrotron, 91190 Saint-Aubin, France
}

(Dated: July 22, 2021)

\begin{abstract}
There is broad consensus that magnetite $\left(\mathrm{Fe}_{3} \mathrm{O}_{4}\right)$ is a promising material for spintronics applications due to its high degree of spin polarization at the Fermi level. However, previous attempts to measure the spin polarization by spin-resolved photoemission spectroscopy have been hampered by the use of low photon energies resulting in high surface sensitivity. The surfaces of magnetite, though, tend to reconstruct due to their polar nature, and thus their magnetic and electronic properties may strongly deviate from each other and from the bulk, dependent on their orientation and specific preparation. In this study we determine the intrinsic - i.e., bulk - spin polarization of magnetite by spin-resolved photoelectron spectroscopy on (111)-oriented thin films, epitaxially grown on $\mathrm{ZnO}(0001)$, with hard $X$-rays, making it a truly bulk-sensitive probe. This becomes possible by using a novel, specially adapted momentum microscope, featuring time-of-flight energy recording and an imaging spin-filter.
\end{abstract}

\section{INTRODUCTION}

Ferrimagnetic magnetite $\left(\mathrm{Fe}_{3} \mathrm{O}_{4}\right)$ has been in the focus of research for many years due to its half-metallic properties, making it an interesting candidate material for spin-electrodes in spintronic devices like, e.g., a DattaDas spin transistor $[1,2]$, all the more as magnetite has a very high Curie temperature of $858 \mathrm{~K}$ [3].

The half-metallic properties can be understood from its inverse-spinel structure (space group $F d \overline{3} m$ ) with tetrahedrally $\left(\mathrm{Fe}_{A}\right)$ and octahedrally $\left(\mathrm{Fe}_{B}\right)$ coordinated iron sublattices. While the $\mathrm{Fe}_{A}$ ions are in the oxidation state $3+, \mathrm{Fe}^{2+}$ and $\mathrm{Fe}^{3+}$ ions are randomly distributed over the $\mathrm{Fe}_{B}$ sublattice [4]. Since there are twice as many $B$ as $A$ sites and the spins of the two sublattices couple antiferromagnetically, the remaining itinerant, uncompensated minority charge carriers on the $\mathrm{Fe}_{B}$ sublattice, give rise to half-metallic behavior.

Since the first spin-resolved photoemission (PE) study on the band structure of natural magnetite single crystals in the 1970s [5], there have been many further attempts to determine the spin polarization. Depending on the type of samples - single crystals and thin films prepared by different deposition techniques - and, specifically, surface orientation and preparation, values for the spin polarization at the Fermi energy $\left(E_{F}\right)$ ranging from $+30 \%$ to $-80 \%$ have been reported over the last 40 years (see Table I) [5-17].

Generally, this surprisingly large scatter of values is rooted in the fact that previous photoemission studies employed low excitation energies in the range from several 10 to several $100 \mathrm{eV}$, resulting in a rather low probing depth of a few atomic layers only. In some cases, photon energies below $10 \mathrm{eV}$ were used but due to the lack of systematic data it is unclear whether, concomitantly, the probing depth is enhanced as was shown for some selected materials [18, 19].

A low probing depth, however, hampers the interpretation of the spin polarization data in the case of magnetite since, due to the specific stacking order of the $\mathrm{Fe}^{2+/ 3+}$ cations and $\mathrm{O}^{2-}$ anions in its inverse-spinel crystal structure, the surfaces are polar which renders them highly unstable with respect to atomic reconstruction [20-22]. This in turn affects the magnetic and electronic properties at the surface which thus may strongly deviate from those of the bulk. In fact, the intrinsic instability of the magnetite (100) and (111) surfaces is known to result in a variety of possible metastable reconstructions [23-25]. Furthermore, the corresponding phases strongly depend on the details of the surface preparation [25], which can cause poor reproducibility in experiment. It is thus not surprising that photoemission has provided a broad range of spin polarization values, with a reliable experimental determination of the true intrinsic bulk value still lacking.

From a theoretical point of view, density-functional theory (DFT) calculations indicate a complete bulk spin polarization of $-100 \%$ at $E_{F}[16,23,24,26-53]$. We note that the few studies explicitly accounting for surface effects $[23,41]$ find the electrons at $E_{F}$ only partially polarized, highlighting again that the properties of surface and bulk strongly differ in magnetite. However, one should bear in mind that the electronic and magnetic properties of magnetite, exhibiting a correlation-induced phase transition, the so-called Verwey transition, may not be 
TABLE I. Overview of the measured spin polarizations $P$ from previous spin-resolved photoemission studies

\begin{tabular}{|c|c|c|c|c|}
\hline Authors & Sample & Method of sample/surface preparation & Photon energy $(\mathrm{eV})$ & $P$ at $E_{F}(\%)$ \\
\hline Kay et al. [6] & $\mathrm{Fe}_{3} \mathrm{O}_{4}(\mathrm{n} / \mathrm{a})$ & reactive sputter deposition on quartz & 4.5 & +30 \\
\hline Kim et al. [7] & $\mathrm{Fe}_{3} \mathrm{O}_{4}(111)$ & oxidation of Fe films grown on W(110) & 40 & +16 \\
\hline Alvarado et al. [5] & $\mathrm{Fe}_{3} \mathrm{O}_{4} \mathrm{nsc}$ & cleaved single crystal & 4.5 & -35 \\
\hline Morton et al. [8] & $\mathrm{Fe}_{3} \mathrm{O}_{4}(100)$ & reactive DC sputtering & 160 & -40 \\
\hline Vescovo et al. [9] & $\mathrm{Fe}_{3} \mathrm{O}_{4}(100)$ & plasma-assisted MBE & 40 & -50 \\
\hline Fonin et al. $[10-12]$ & $\mathrm{Fe}_{3} \mathrm{O}_{4}(100)$ & MBE & 58 & -55 \\
\hline Huang et al. [13] & $\mathrm{Fe}_{3} \mathrm{O}_{4}(100)$ & MBE & 450 & -55.5 \\
\hline Alvarado et al. [14] & $\mathrm{Fe}_{3} \mathrm{O}_{4} \mathrm{nsc}$ & cleaved single crystal & 5.2 & -65 \\
\hline Tobin et al. $[15]$ & $\mathrm{Fe}_{3} \mathrm{O}_{4}(100)$ & reactive DC sputtering & 160 & -65 \\
\hline Wang et al. $[16]$ & $\mathrm{Fe}_{3} \mathrm{O}_{4}(100)$ & epitaxial grown on $\mathrm{MgO}(001)$ & 4.65 & -72 \\
\hline Dedkov et al. $[17]$ & $\mathrm{Fe}_{3} \mathrm{O}_{4}(111)$ & oxidation of Fe films grown on W(110) & 21.2 & -80 \\
\hline Fonin et al. $[10,12]$ & $\mathrm{Fe}_{3} \mathrm{O}_{4}(111)$ & oxidation of Fe films grown on W(110) & 21.2 & -80 \\
\hline
\end{tabular}

DC: Direct current, MBE: Molecular beam epitaxy, nsc: Natural single crystal

adequately captured by DFT calculations since correlations are treated only statically on a mean-field level. Furthermore, only groundstate properties are considered in DFT instead of the photoemission process itself. Alvarado and Bagus [54] early on did consider the actual photoemission process in a single ion model for ferrites, accounting for the spin coupling between the photoelectron and the final state of the correlated $3 d^{n-1}$-shell left behind. This approach, describing the atomic limit, also results in a negative spin polarization with the emission predominantly that of minority spin electrons, due to the high-spin final state for more than half-filled $3 d$ shells, but with a significantly lower value of $-66.7 \%$. Clearly, this model completely neglects band structure effects. An overview of previous theoretical studies and the predicted spin polarizations $P$ therein is shown in Table II.

To settle the issue of the intrinsic spin polarization of magnetite, truly bulk-sensitive spin-resolved PE has to be performed to rule out any surface effects. Previous spin- and angle-integrated measurements of the valence band showed still significant differences in the spectra for the (111) and (100) surface orientations at photon energies as high as $\approx 700 \mathrm{eV}$ [55]. Therefore, to really probe bulk properties, photon energies in the hard X-ray regime have to be used. This was previously hampered by the notoriously low photoionization cross sections in the hard $\mathrm{X}$-ray regime in combination with the poor efficiency for Mott scattering, which for a long time was the basic design principle of photoemission spin detectors. Therefore, new routes have to be followed [56-59] to enable bulksensitive, spin-resolved photoemission experiments. In our study, this was accomplished by using a newly developed time-of-flight (ToF) $k$-space microscope-equipped with a spin-filter crystal-, that operates in the hard Xray regime and was installed at a dedicated synchrotron beamline. Thus, this instrument allows for simultaneous energy-, spin-, and $k$-resolved mapping of the photoelec- trons excited by each single photon pulse with unprecedented efficiency.

\section{SAMPLE GROWTH AND CHARACTERIZATION}

High quality $\mathrm{Fe}_{3} \mathrm{O}_{4}(111)$ films of $30 \mathrm{~nm}$ thickness were grown on $\mathrm{ZnO}(0001)$ substrates by molecular beam epitaxy as described in Ref. 60. Iron was evaporated from a high temperature effusion cell in an oxygen atmosphere of about $3.5 \cdot 10^{-6}-7.0 \cdot 10^{-7}$ mbar while the substrate was kept at a temperature of $400^{\circ} \mathrm{C}$. These growth parameters are crucial since there are many stable iron oxide compounds beside magnetite [61].

In a previous study we have already shown by measuring with a superconducting quantum interference device (SQUID) that our magnetite films show a spinreorientation transition at $128 \mathrm{~K}$, which is known to lie approximately $10 \mathrm{~K}$ above the actual Verwey transition temperature and is comparable to those observed in bulk single crystals [60]. Furthermore, X-ray diffraction (XRD) $\omega-2 \theta$-scans and rocking curves exhibit nearly completely relaxed growth on $\mathrm{ZnO}$ and single crystal quality, as indicated by a full width at half maximum of the rocking curves of only $0.04^{\circ}[60,62]$.

To further check the crystalline quality of the films, in situ low-energy electron diffraction (LEED) was performed [see Fig. 1(a)]. The sharp hexagonal $2 \times 2$ LEED pattern, showing the reciprocal primitive cells of the oxygen (red) and iron (blue) sublattices, confirms the formation of an atomically long-range ordered $\mathrm{Fe}_{3} \mathrm{O}_{4}(111)$ surface [63].

A big advantage of the ToF $k$-space microscope used in this study (see Appendix) is its capability to acquire constant energy maps "at one shot", i.e., without tilting the sample. This provides the possibility to record hard X- 
TABLE II. Theoretical calculations based on various approximations and their predicted bulk spin polarization $P$

\begin{tabular}{|c|c|c|c|}
\hline Authors & Year & Implementation/Approximation & $P$ at $E_{F}(\%)$ \\
\hline Alvarado and Bagus [54] & 1978 & single ion model & $-66.7^{* *}$ \\
\hline Yanase and Siratori $[26]$ & 1984 & SC-APW & -100 \\
\hline de Groot and Buschow [27] & 1986 & GGA(SC-ASW) & -100 \\
\hline Zhang and Satpathy [28] & 1991 & LSDA(LMTO-ASA) & -100 \\
\hline Pénicaud et al. [29] & 1992 & GGA(SC-ASW) & -100 \\
\hline Anisimov et al. [30] & 1996 & LSDA & -100 \\
\hline Yanase and Hamada [31] & 1999 & FP-APW & -100 \\
\hline Antonov et al. $[32,33]$ & 2001, 2003 & $\mathrm{LSDA}+\mathrm{U}$ & -100 \\
\hline Jeng et al. $[34-36]$ & $2002,2004,2006$ & $\mathrm{LSDA}(\mathrm{APW}, \mathrm{ASW}, \mathrm{LMTO}) / \mathrm{LDA}+\mathrm{U}$ & -100 \\
\hline Szotek et al. [37] & 2003 & SIC-LSDA & -100 \\
\hline Leonov et al. [38] & 2004 & $\mathrm{LSDA}+\mathrm{U}(\mathrm{TBLMTO})$ & -100 \\
\hline Madsen and Novák [39] & 2005 & LDA+U(FLL-DCC) & -100 \\
\hline Pentcheva et al. [23] & 2005 & GGA(FP-APW) & $-40^{*}$ \\
\hline Pinto and Elliott [40] & 2006 & $\mathrm{GGA}+\mathrm{U}(\mathrm{PAW})$ & -100 \\
\hline Zhu et al. [24] & 2006 & $\mathrm{LDA}+\mathrm{U}$ & -100 \\
\hline Eodziana [41] & 2007 & $\mathrm{GGA}+\mathrm{U}$ & $>-100^{*}$ \\
\hline Piekarz et al. $[42,43]$ & 2007,2010 & $\operatorname{GGA}(+\mathrm{U})$ & -100 \\
\hline Rowan et al. [44] & 2009 & GGA & -100 \\
\hline $\mathrm{Yu}$ et al. [45] & 2012 & $\mathrm{GGA}+\mathrm{U}$ & -100 \\
\hline Arras et al. [46] & 2013 & $\mathrm{LSDA}+\mathrm{U}(\mathrm{FP}-\mathrm{APW})$ & -100 \\
\hline Wang et al. [16] & 2013 & $\mathrm{GGA}+\mathrm{U}$ & -100 \\
\hline Noh et al. [47] & 2014 & $\mathrm{GGA}+\mathrm{U}$ & -100 \\
\hline Schmitz et al. [48] & 2014 & $\mathrm{GGA}+\mathrm{U}$ & -100 \\
\hline Liu et al. $[49,50]$ & 2017,2019 & GGA+U/SCC-DFTB & -100 \\
\hline Mounkachi et al. [51] & 2017 & SIC-LSDA & -100 \\
\hline Chen et al. [52] & 2018 & $\operatorname{GGA}(+\mathrm{U})$ & -100 \\
\hline Sai Gautam and Carter [53] & 2018 & $\operatorname{SCAN}(+\mathrm{U})(\mathrm{PAW})$ & -100 \\
\hline
\end{tabular}

SC/FP-APW: Self-consistent/full-potential augmented-plane-wave, SC-ASW: Self-consistent augmented spherical waves, FLL-DCC: Fully localized limit - double counting corrected, GGA: Generalized gradient approximation, (TB)LMTO(-ASA): (Tight binding) linear muffin-tin orbital (atomic spheres approximation), PAW: Projector augmented wave, (SIC)-L(S)DA: (Self-interaction corrected) local (spin) density approximation, SCAN: Strongly constrained and appropriately normed, SCC-DFTB: Self-consistent charge density functional tight binding

${ }^{*}$ Calculations were done explicitly for the surface of $\mathrm{Fe}_{3} \mathrm{O}_{4}(001)$.

** Represents the photoelectron spin polarization of the whole $\mathrm{Fe}^{2+} 3 d$ shell.

ray photoelectron diffraction $(\mathrm{hXPD})$ patterns with large probing depths in only a few minutes, even on samples that have been previously exposed to air. While LEED essentially yields information on the structural quality and symmetry of the very surface, hXPD, being based on the forward scattering of photoelectrons, gives detailed information about the geometrical structure of subsurface atomic layers reaching far into the bulk [64].

Figure 1(b) displays on the left side the measured hXPD patterns for the Fe $3 p$ and O $2 p$ core levels along with simulations on the right side, based on the manybeam dynamical theory of electron diffraction [65]. The hXPD patterns of $\mathrm{Fe} 3 p$ and $\mathrm{O} 2 p$ were measured with excitation energies of $5204 \mathrm{eV}\left(E_{k i n}=5150 \mathrm{eV}\right)$ and
$5000 \mathrm{eV}\left(E_{k i n}=4993 \mathrm{eV}\right)$, respectively. The simulations were carried out at the same energies. The good agreement of theory and experiment confirms the good structural quality of the sample also in the bulk and supports the claim of a truly bulk-sensitive measurement at these photon energies.

In addition, immediately after epitaxial growth within the same vacuum system XPS using $\mathrm{Al} \mathrm{K}_{\alpha}$ radiation and an Omicron EA 125 hemispherical deflection analyzer (HDA) was performed in our home lab in Würzburg. In Fig. 2(a) the Fe $2 p$ core level spectrum is shown, exhibiting the characteristic line shape of magnetite as originates from the $\mathrm{Fe}^{2+}$ and $\mathrm{Fe}^{3+}$ ions of this mixed-valency compound including corresponding charge-transfer (CT) 
(b)

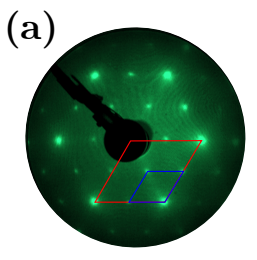

Experiment

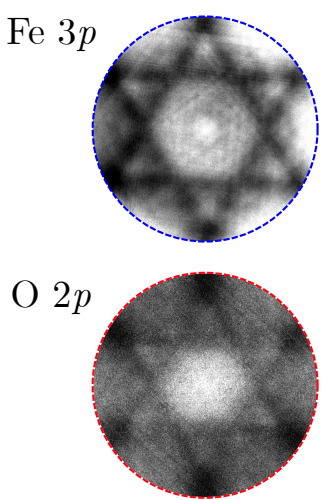

Simulation

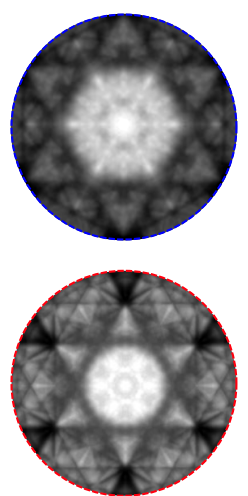

FIG. 1. (a) LEED pattern $(98.5 \mathrm{eV})$ of a freshly grown magnetite film, showing the reciprocal primitive cells of the oxygen (red) and iron (blue) sublattices. (b) hXPD patterns of the Fe $3 p\left(E_{k i n}=5150 \mathrm{eV}\right)$ and $\mathrm{O} 2 p\left(E_{k i n}=4993 \mathrm{eV}\right)$ core levels: experimental data on the left and calculated diffraction patterns on the right are in good agreement, confirming the excellent structural quality of the film and also supporting the claim of bulk sensitivity of the measurements. The hXPD patterns cover an angle range of $\pm 9^{\circ}$.

satellites and is markedly distinct from other iron oxides [61]. In (b) the XPS spectrum of the valence band is compared to an ex situ spin-integrated photoemission spectrum of the same sample recorded with the ToF microscope at the hard X-ray beamline P22 at the synchrotron PETRA III, DESY (Hamburg, Germany) [66]. Due to the better energy resolution of the ToF instrument in combination with the highly brilliant synchrotron radiation, the Fe $3 d$ states at $E_{F}$ are less broadened and hence appear more pronounced than in the spectrum taken in our home lab. Otherwise, the different spectral lineshapes can be explained by the relative change in the photoionization cross sections of the $\mathrm{O} 2 p$ and Fe $3 d$ states that are hybridized with each other. Nevertheless, for both photon energies the predominant contribution of the Fe $3 d$ states at the Fermi level remains clearly distinguishable from the mainly $\mathrm{O} 2 p$-derived valence band spectral weight at higher binding energies.

Furthermore, X-ray magnetic circular dichroism (XMCD) has been measured on an $\mathrm{LN}_{2}$ cooled film in total electron yield mode at the TEMPO beamline (SOLEIL, France) to establish magnetic ordering. The blue and red spectra in Fig. 3 reflect the absorption measured at the Fe $L_{2,3}$ edge $(2 p \rightarrow 3 d$ transition) with different helicity (parallel $\sigma_{+}$and antiparallel $\sigma_{-}$with respect to the in-plane magnetization). Both curves were normalized by the mirror current $I_{0}$, which is a measure for the incident photon flux. The absorption spectra and the resulting XMCD signal $\left[\left(\sigma_{+}-\sigma_{-}\right) \cdot 2\right.$, corrected for the experimental geometry with the beam incidence under $45^{\circ}$ relative to the surface normal], which is shown as green dashed line in Fig. 3 [67], are in very good agreement with other theoretical and experimental studies on mag-

(a)
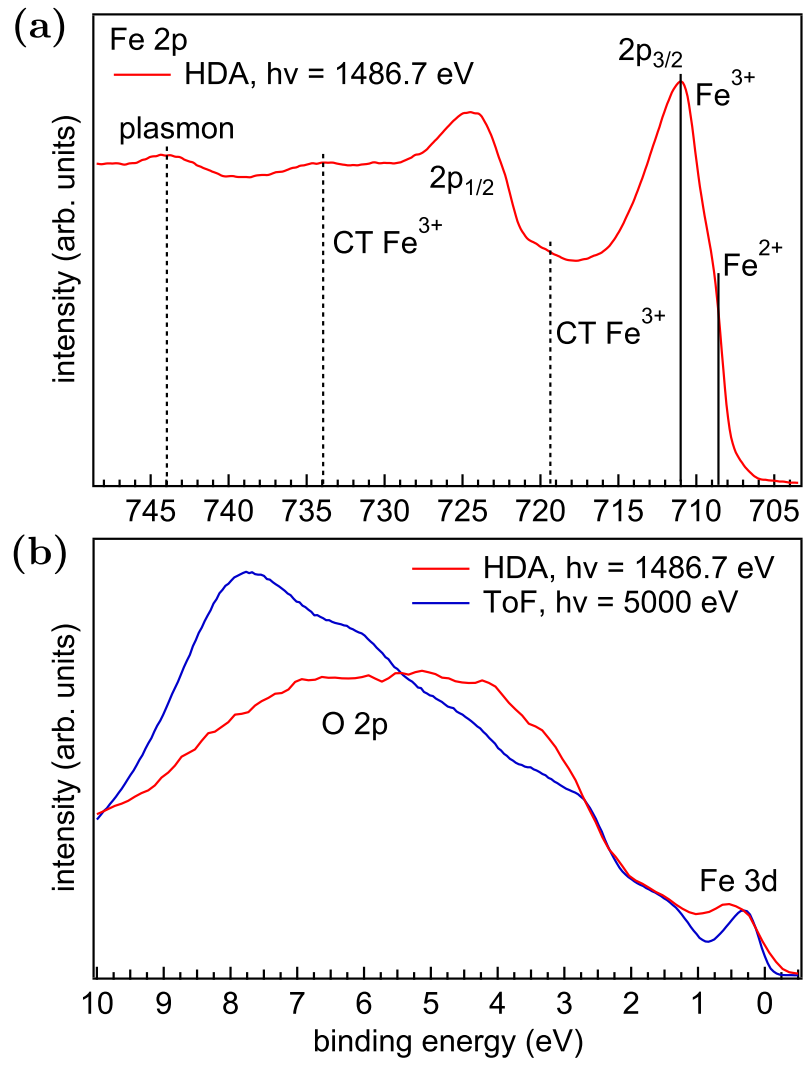

FIG. 2. (a) Fe $2 p$ core level spectrum measured with $\mathrm{Al} \mathrm{K}_{\alpha}$ radiation, showing $\mathrm{Fe}^{2+}$ and $\mathrm{Fe}^{3+}$ contributions due to the mixed valency of magnetite as well as charge-transfer (CT) satellites. (b) Hard X-ray (blue) and lab-based (red) valence band spectra of the same magnetite film. The Fe $3 d$ states at $E_{F}$ are clearly distinguishable from the O $2 p$ valence band.

netite thin films [68-72]. The three peaks in the XMCD signal thereby represent the B-site Fe ions (a and c) and the $\mathrm{Fe}^{3+}$ ions on the A-site (b).

To summarize this section, SQUID and XRD measurements from a previous study as well as LEED, hXPD, spin-integrated photoemission, and XMCD establish the high quality of the $\mathrm{Fe}_{3} \mathrm{O}_{4}$ films in terms of bulk and surface crystallinity, magnetic ordering, and stoichiometric composition. They hence can be regarded as representative for bulk properties similar to single crystals.

\section{SPIN-RESOLVED PHOTOEMISSION}

In our photoemission experiment we use the spindependent reflectivity at the surface of an iridium single crystal to gain information on the photoelectron spin (see Appendix and references therein for detailed information). The strongest spin-sensitivity is achieved if the photoelectrons are decelerated to a kinetic energy of about $12.5 \mathrm{eV}$ when impinging on the spin-filter crystal, making the spin-dependent scattering extremely surface sensitive. Therefore, prior to the spin-resolved measure- 


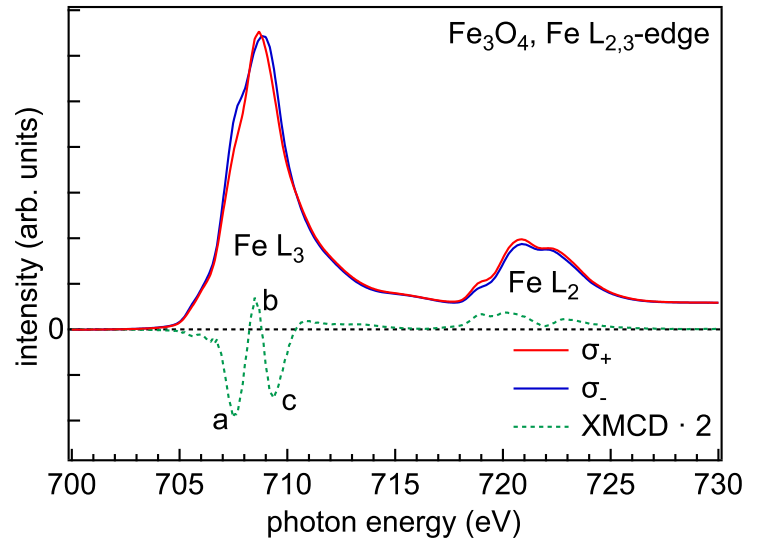

FIG. 3. X-ray magnetic circular dichroism measured at the Fe $L_{2,3}$ absorption edge. The blue and red curves reflect the absorption for different helicity $\sigma_{+}$and $\sigma_{-}$, respectively. Both curves were normalized by the mirror current $I_{0}$. The resulting XMCD signal is shown as green dashed line (multiplied by 2$)$.

ments the surface of the iridium spin-filter crystal was freshly prepared by several high temperature flashes in oxygen atmosphere to get rid of surface contaminants like carbon and nitrogen compounds, which can significantly decrease the reflectivity and spin asymmetry of the reflected intensity [73]. The low scattering energy concentrates all reflected intensity into only a few diffraction spots, being favorable for the Figure-of-Merit of spin detection using the specular (0,0)-reflex.

Although the microscope allows for simultaneous spinand $\mathrm{k}$-resolved mapping of the photoelectrons, due to the weak dispersion of the Fe $3 d$ bands, their momentum dependence cannot be resolved [74]. Therefore, the spinresolved data will be integrated over the area covered by the spin-filter crystal $\left(\approx 7 \AA^{-1}\right.$ in $k_{x^{-}}$and $\approx 4 \AA^{-1}$ in $k_{y}$-direction), granting a higher signal-to-noise ratio due to higher count rates. The sample was cooled down to a temperature of $\approx 30 \mathrm{~K}$ in order to avoid magnetic fluctuations and - as a positive side effect - to reduce the DebyeWaller factor that determines the strength of direct transitions in relation to phonon-induced thermal scattering contributions. The cooled sample was then magnetized in-plane $\left(I^{+}\right)$with a permanent magnet. The applied field amounted to 1 Tesla parallel to the film and to the quantization axis of the spin detector, large enough to drive the sample into saturation. The valence band was then measured in remanence with an excitation energy of $\mathrm{h} \nu=5.0 \mathrm{keV}[75]$, leading to a photoelectron inelastic mean free path of $\approx 70 \AA[76]$ and an energy resolution of $\approx 620 \mathrm{meV}$ - using a $\mathrm{Si}(111)$ monochromator crystal pair for maximum photon flux. Then the sample was magnetized in the opposite direction $\left(I^{-}\right)$and measured again. Figure 4(a) shows the spectra for the two opposite in-plane magnetization directions for the relevant energy range from $E_{F}$ up to $5 \mathrm{eV}$. The inset shows the entire acquired range up to $10 \mathrm{eV}$ with no visible difference in the spectral shape for binding energies above $3 \mathrm{eV}$.

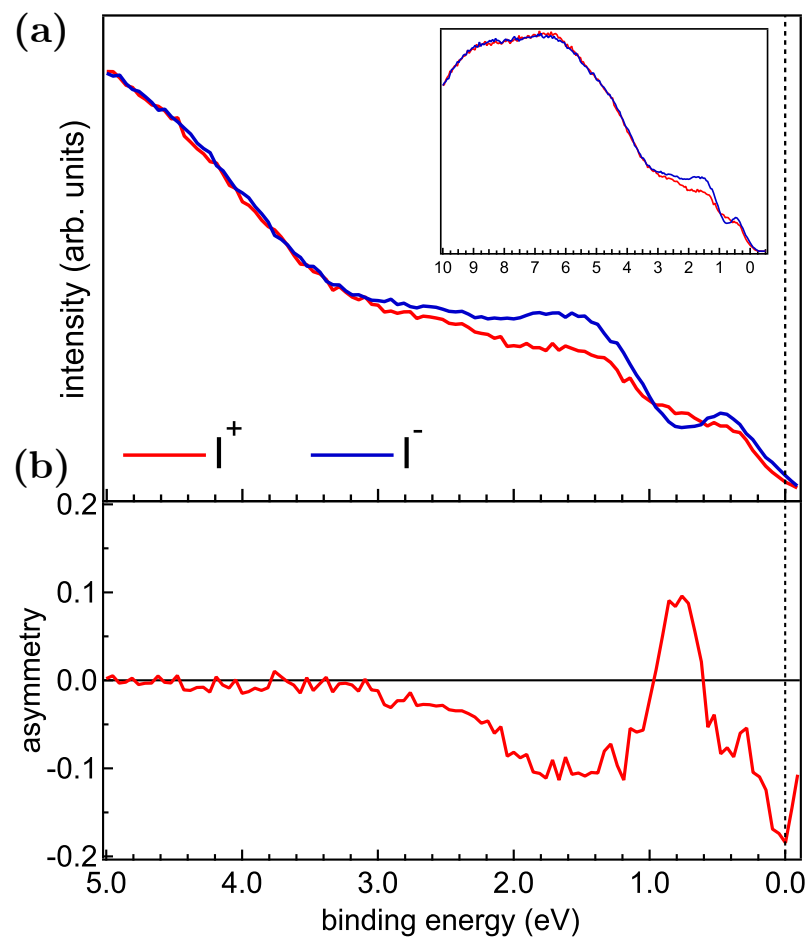

FIG. 4. (a) Valence band spectra measured with an excitation energy of $\mathrm{h} \nu=5.0 \mathrm{keV}$ for two opposite in-plane magnetizations denoted by $I^{+}$and $I^{-}$. (b) Energy dependent asymmetry $A$, calculated with Eq. 1 .

In contrast, clear differences between the two magnetizations are seen for smaller binding energies. The resulting intensity asymmetry $A$, defined by

$$
A=\frac{I^{+}-I^{-}}{I^{+}+I^{-}},
$$

is plotted in Fig. 4(b). It is zero above $3 \mathrm{eV}$ where the O $2 p$ valence band dominates. For smaller binding energies and with predominantly Fe $3 d$ states contributing, the asymmetry first becomes negative and switches sign between $0.95 \mathrm{eV}$ and $0.60 \mathrm{eV}$ with a maximum at $0.80 \mathrm{eV}$. Closer to the Fermi level the asymmetry turns negative again and assumes a maximum negative value at $E_{F}$.

The conversion of the measured asymmetry $A$ into an actual spin polarization $P$ involves the Sherman function, being a measure of the spin-sensitivity of the used spinfilter crystal:

$$
P=\frac{N^{\uparrow}-N^{\downarrow}}{N^{\uparrow}+N^{\downarrow}}=\frac{1}{S} \frac{I^{+}-I^{-}}{I^{+}+I^{-}},
$$

where $N^{\uparrow}$ and $N^{\downarrow}$ are the total photoelectron numbers with spin $\uparrow$ and $\downarrow$, respectively. It is important to realize that in our specific experimental approach the explicit energy dependence of the Sherman function $S=S(E)$ is required, in contrast to conventional spin-detectors operated at a fixed energy only. Employing ToF energy 
recording, all photoelectrons in the measured energy window are reflected off the spin-filter crystal essentially simultaneously, i.e., at different scattering energies. For the data in Fig. 4 the electron optics was set in such a way that photoelectrons originating from the maximum of the Fe $3 d$ peak at $\approx 0.5 \mathrm{eV}$ reach the spin-filter crystal at a kinetic energy where its Sherman function assumes its maximum $(\approx 12.5 \mathrm{eV})$ [77]. Consequently, electrons at higher (lower) binding energy get scattered at lower (higher) kinetic energy, with subsequently different spin efficiency as captured by $S(E)$ and hence necessitating a careful calibration of the Sherman function.

Such a calibration is best achieved on a material system with known spin polarization. Here we chose EuO whose $\mathrm{Eu} 4 f$ states have been demonstrated to display a spin polarization of $100 \%$ [78, 79]. Based on this value we measured the experimental spin asymmetry of the $4 f$ states of a thin $\mathrm{EuO}$ film [79] for scattering energies between $9.5 \mathrm{eV}$ and $13.2 \mathrm{eV}$, and calculated the associated values of the Sherman function. Figure 5 depicts the corresponding data points together with a spline interpolation. The Sherman function assumes its maximum around $12.5 \mathrm{eV}$ while at lower kinetic energies, i.e., higher binding energies, $S(E)$ is significantly decreasing and even changes its sign at about $10.9 \mathrm{eV}$.

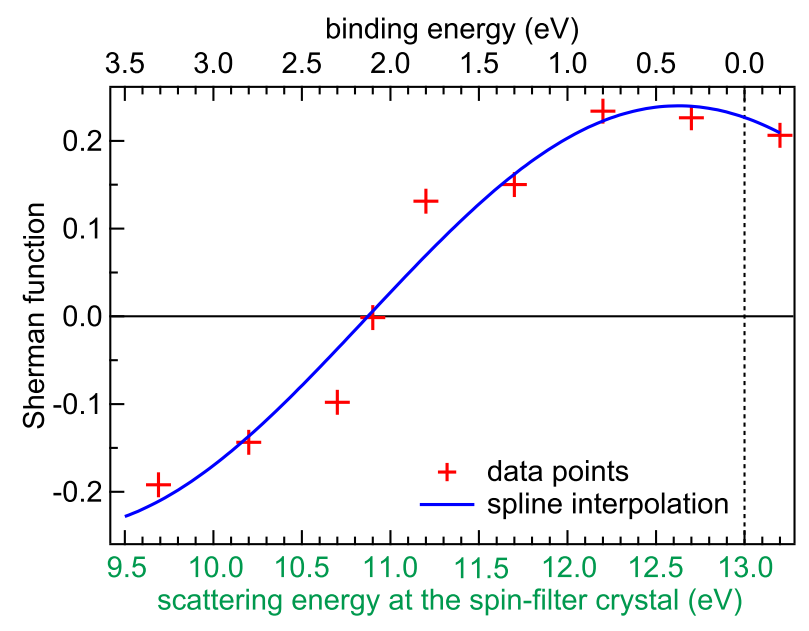

FIG. 5. Energy dependent Sherman function $S(E)$, calibrated using the known $100 \%$ spin polarization of the Eu $4 f$ states of an EuO thin film. The bottom axis refers to the actual scattering energy at the spin-filter crystal. In the top axis these values are converted to a binding energy scale, as adapted to the spin-resolved magnetite spectra of Fig. 4.

Based on this calibration of the Sherman function we finally determine the energy-dependent spin polarization $P(E)$ of our magnetite film from Eq. 2. The result is shown in Fig. 6. As predicted by theory, the spin polarization at $E_{F}$ is negative. With higher binding energies, the absolute value of the spin polarization decreases and switches sign at $0.60 \mathrm{eV}$. It exhibits a maximum with further increasing binding energies and becomes negative

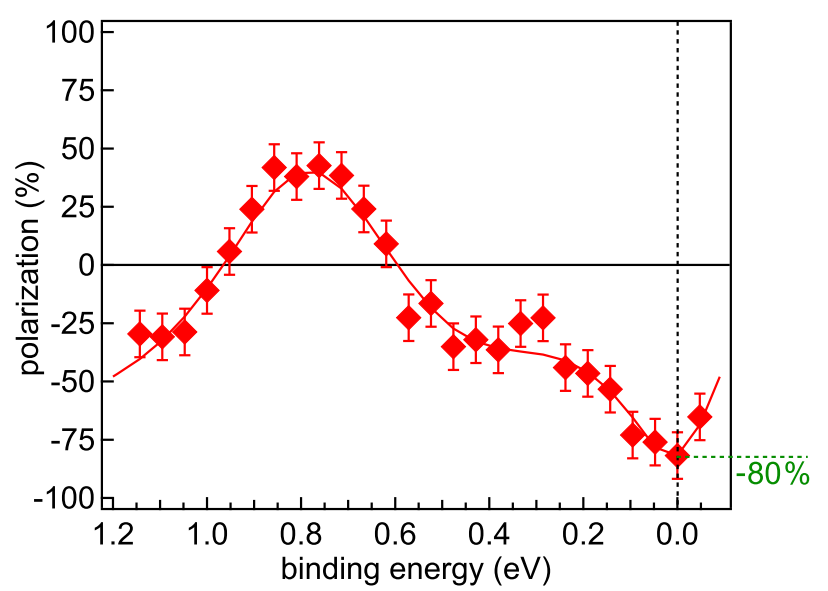

FIG. 6. Spin polarization of the magnetite film, calculated with Eq. 2 (the line serves as guide to the eye). It is negative at $E_{F}=0 \mathrm{eV}$ with a value of $-80 \%$. Between $0.60 \mathrm{eV}$ and $0.95 \mathrm{eV}$, there is a sign change of the polarization. The error bars denote the statistical error.

again at $0.95 \mathrm{eV}$. Since the Sherman function has a zero at about $10.9 \mathrm{eV}$, corresponding to a binding energy of $2.1 \mathrm{eV}$ in our study, the spin polarization unphysically diverges around this energy, and hence we focus in Fig. 6 on smaller binding energies from $1.2 \mathrm{eV}$ up to the Fermi level, where the Sherman function does not vary strongly and the polarization is not affected by the zero in $S$. For binding energies above $3 \mathrm{eV}$ the polarization becomes zero (see Fig. 4).

To give a quantitative estimate of the spin polarization at $E_{F}$ from the measured asymmetry, we first point out that the measurements were performed at $\approx 30 \mathrm{~K}$, i.e., below the Verwey temperature, at which a metal-insulator transition takes place and a small gap of about $50 \mathrm{meV}$ with respect to $E_{F}$ opens [55]. So, strictly speaking, there are no states at $E_{F}$ and thus no defined spin polarization. However, the finite energy resolution of about $600 \mathrm{meV}$ in our experiment transfers detectable spectral weight to the Fermi level, resulting in a finite polarization signal. The experimental spin polarization at the Fermi level $P\left(E_{F}\right)$ can now be determined from Fig. 6 to be $-80 \%$ (dashed green line).

The statistical error for the asymmetry shown in Fig. 4(b) is estimated from the count rates $I^{+}$and $I^{-}$ in a $50 \mathrm{meV}$ energy interval near $E_{F}$ for both magnetization directions, $\Delta A=\sqrt{4 I^{+} I^{-} / I^{3}}=0.012$ (with $\left.I=I^{+}+I^{-}\right)$. The statistical error of the Sherman function related to the calibration measurement is in the same order of magnitude, $\Delta S=0.013$ (see Fig. 5). The statistical error of the polarization value results from the sum of the relative errors, $\Delta P / P=\Delta A / A+\Delta S / S$. Near the Fermi energy the statistical error amounts to $\Delta P / P=0.13$ and $\Delta P=0.104$. As additional systematic errors, we take into account (i) reduced remnant magnetization compared to the intrinsic magnetization, and (ii) finite energy resolution. (i) As the sample was measured in remanence, the magnetization $M_{r}$ and thereby the spin 
polarization could be lower than the intrinsic magnetization $M_{s}$. Previous SQUID magnetometry on similarly prepared samples result in $M_{r} / M_{s}=0.64$ [60]. Therefore, the intrinsic spin polarization could be larger than the measured value. (ii) Due to the specific shape of the energy-dependent polarization data in Fig. 6 (with a maximal positive spin polarization at $0.8 \mathrm{eV}$, a sign change at $0.6 \mathrm{eV}$, and a maximal negative spin polarization at the Fermi energy), a finite energy resolution tends to reduce the measured spin polarization at $E_{F}$. Therefore, the measured spin polarization at $E_{F}$ represents a lower limit of its true absolute value and strongly points to a full spin polarization at $E_{F}$ of $-100 \%$, supporting the assumption of magnetite being a half-metallic ferromagnet. Considering both statistical and systematical errors, the spin polarization value at $E_{F}$ amounts to $P\left(E_{F}\right)=-80_{-20}^{+10} \%$.

Finally, the spin-resolved density of states (spin-DOS) can be inferred from Eq. 2 with $N_{\text {tot }}=N^{\uparrow}+N^{\downarrow}$ :

$$
N^{\uparrow \downarrow}=\frac{1}{2}(1 \pm P) N_{t o t} .
$$

The resulting majority and minority spin-densities are shown in Fig. 7 (red and blue triangles). Also shown are the results of DFT calculations by Piekarz et al. [42]performed explicitly for the low temperature phase of magnetite with the monoclinic P2/c symmetry [80, 81] and including strong correlation effects on the GGA $+\mathrm{U}$ level-, without (solid lines) and with (dashed lines) the experimental broadening taken into account. While the experimental spin-densities overall resemble the theoretical ones, there are also notable quantitative differences. It has been argued that, in general, magnetite should be considered a correlated electron system, casting a description by DFT and its findings of half-metallicity into doubt [13]. Furthermore, the excitation of spin waves $[10,17,82,83]$ and the formation of polarons [16], which both are not captured by DFT calculations, were put forward in previous experimental studies to explain a smaller spin polarization observed in experiment than predicted by DFT. Such effects also will affect the detailed experimental lineshapes in the near- $E_{F}$ region.

\section{CONCLUSIONS}

In this study we have measured the bulk spin polarization of magnetite by spin-resolved photoelectron spectroscopy. For these experiments, we have utilized a new generation of photoemission instrumentation. It is featuring time-of-flight energy recording in combination with imaging spin-filtering and adapted for operation with hard X-rays at a dedicated synchrotron beamline to strongly enhance the probing depth. With this setup, it has become possible to overcome the notorious problem of ill-defined magnetite surfaces from which previous lowenergy spin-resolved photoemission studies suffered. The measured spin polarization of $-80_{-20}^{+10} \%$ strongly points

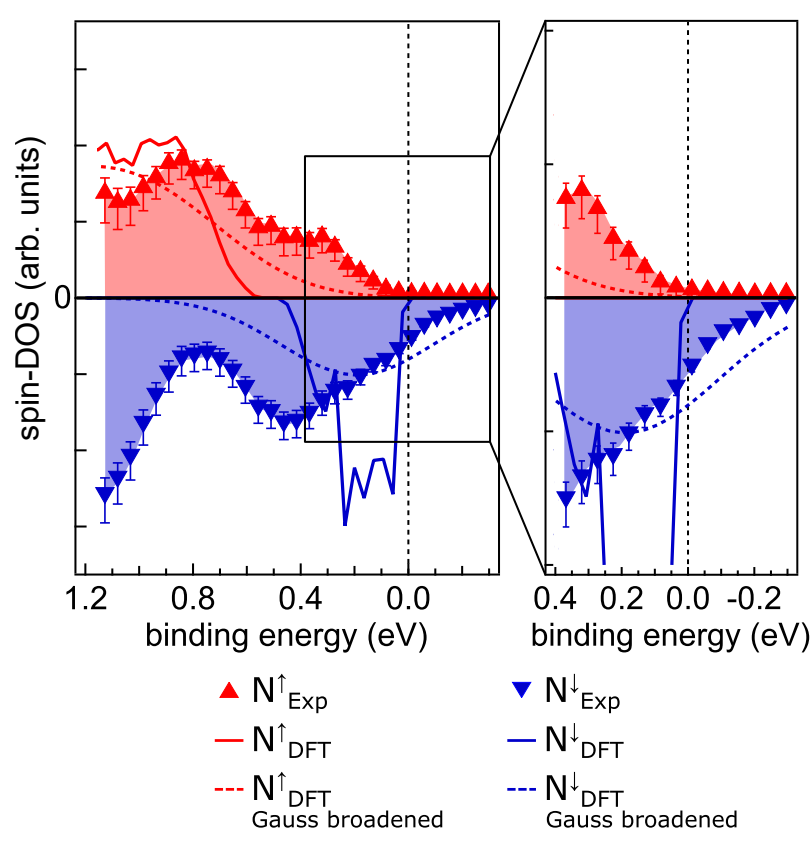

FIG. 7. Majority and minority spin-DOS (red and blue triangles, respectively) for $\mathrm{Fe}_{3} \mathrm{O}_{4}$, calculated from the results of Fig. 4 using Eq. 3 with error bars denoting the statistical error. Mostly minority spin states are located at $E_{F}$. The spin-DOS overall resembles those from DFT calculations (solid lines) in Ref. 42, especially upon convolution with the instrumental resolution (dashed lines).

to a full bulk spin polarization at $E_{F}$, fully compatible with theoretical predictions from density-functional theory that magnetite is a half-metallic ferromagnet.

From an experimental view, further advancements in instrumentation, regarding both electron and photon energy resolution, will make it possible to narrow down the experimental uncertainty for the spin polarization of magnetite further [84]. This will have to be matched by improved theoretical approaches, taking properly into account both the itinerancy of the minority charge carriers at the Fermi energy as well as their correlations and coupling to the lattice degrees of freedom. Nevertheless, our present results point to a full bulk spin polarization near $E_{F}$, that originates from spin-down charge carriers, and thereby provide strong experimental support to the theoretical view of magnetite as a half-metallic ferromagnet.

\section{ACKNOWLEDGMENTS}

We thank Fausto Sirotti (Laboratoire de Physique de la Matière Condensée, Palaiseau, France) for fruitful discussions about the X-ray absorption measurements.

This work was supported by the Bundesministerium für Bildung und Forschung (BMBF) through the project EffSpin-HAXPES (project numbers 05K16WWA and 05K16UMC). We acknowledge further support by the Deutsche Forschungsgemeinschaft (DFG, German Re- 
search Foundation) through the Würzburg-Dresden Cluster of Excellence on Complexity and Topology in Quantum Matter ct.qmat (EXC 2147, Project ID 390858490), the Collaborative Research Center SFB 1170 ToCoTronics (Project ID 258499086) in Würzburg, and the Transregional Collaborative Research Center TRR 173 Spin + X (Project ID 268565370) in Mainz. A.W. acknowledges support by Polish National Science Centre (NCN, grant no. 2020/37/B/ST5/03669).

The Deutsches Elektronen-Synchrotron DESY (Hamburg, Germany) is gratefully acknowledged for beamtime under proposals no. I-20181063, I-20181092, and I-20190546, and the SOLEIL Synchrotron (Saint-Aubin, France) for beamtime under proposal no. 99200077.

\section{Appendix: Spin-resolved time-of-flight $k$-space microscopy}

Spin-resolved photoemission poses very high demands on the experimental setup since the discrimination of spin orientations, on whichever microscopic mechanism it is based, in general has a very low efficiency compared to spin-integrated photoemission. Hence, it is important to generate and count as many photoelectrons as possible. One of the most common setups to date is the combination of a hemispherical deflection analyzer (HDA) with a Mott detector [85]. However, due to the tremendous decrease of the photoabsorption cross sections with increasing photon energies such instruments are of limited use in the hard X-ray regime - especially when studying valence bands - , since they only offer single-channel detection. To benefit from the multichannel capabilities of modern energy analyzers and detectors, a new type of spin detector - known from the soft X-ray and vacuum ultraviolet (VUV) spectral range $[77,86]$ —was adopted for hard X-rays several years ago, making use of an iridium single crystal as spin-filter [77]. Although the reflectivity of the spin-filter crystal is in the range of only a few percent, the spin-resolved, parallel acquisition of photoelectrons makes such type of instruments superior to spectrometers equipped with a Mott detector.

As a further improvement-instead of employing the $2 \mathrm{D}$ recording scheme of a HDA, which requires entrance and exit slits to obtain a decent energy resolution at the sacrifice of electron count rates-, the ToF method was introduced to discriminate all electrons emitted from a single photon pulse with respect to their energy enabling $3 \mathrm{D}\left(k_{x}, k_{y}, E_{k i n}\right)$ recording and thus profit from much higher count rates. Based on this technique combined with a newly designed extractor lens, optimized for low aberrations at very high excitation energies, a highly efficient $k$ - and spin-resolved ToF microscope was set up at P22, DESY (Hamburg, Germany) [66]. A sketch of the principal components is illustrated in Fig. 8. The spinfilter crystal is retractable, allowing for two brancheseach with its own delay-line detector. One for measuring spin- and $k$-resolved with a scattering angle of $56^{\circ}$ with respect to the crystal surface normal, and the other one for high-intensity spin-integrated $k$-mapping. Using the 40 bunch mode of PETRA III with a bunch separation of $192 \mathrm{~ns}$, the energy and momentum resolution of the microscope for the measurements shown here amounted to $40 \mathrm{meV}$ and $0.025 \AA^{-1}$, respectively, which represent typical values for this kind of instrument $[87,88]$. The key factor making the present experiment feasible is the increased Figure-of-Merit of the spin-filter approach shown in the inset. A comparison of quantitative values for different spin-filter concepts is given in Fig. 28 of Ref. 89.

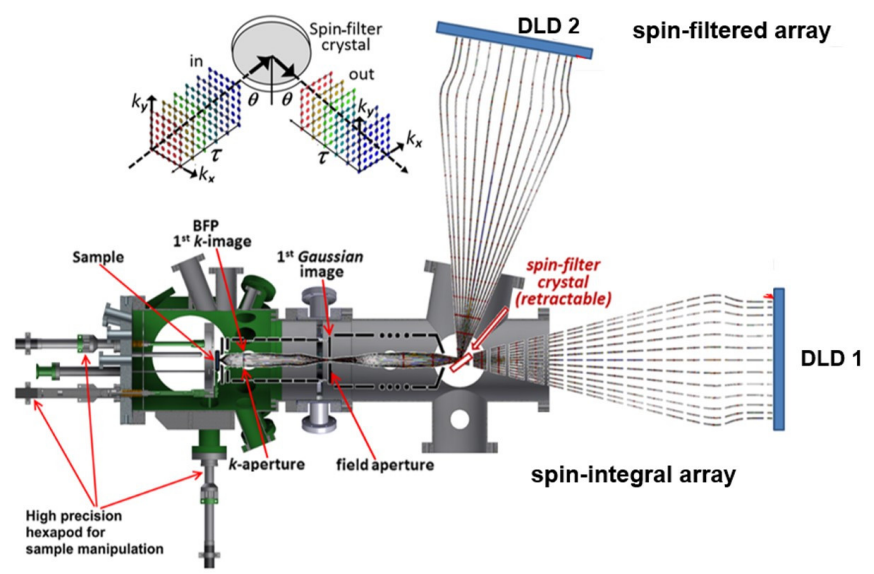

FIG. 8. Design (side view) and principal operation of the newly set-up microscope at beamline P22, DESY. Notice that the spin-filter also preserves the energy information encoded in the time-of-flight signal (see inset), resulting in a $3 \mathrm{D}\left(k_{x}\right.$, $\left.k_{y}, E_{k i n}\right)$ recording scheme.
[1] S. Datta and B. Das, Appl. Phys. Lett. 56, 665 (1990).

[2] R. Winkler and M. Oestreich, Physik Journal 3, 39 (2004).

[3] M. Ziese, Rep. Prog. Phys. 65, 143 (2002).

[4] M. E. Fleet, Acta Cryst. B 37, 917 (1981).

[5] S. F. Alvarado, W. Eib, F. Meier, D. T. Pierce, K. Sattler, H. C. Siegmann, and J. P. Remeika, Phys. Rev. Lett. 34, 319 (1975).

[6] E. Kay, R. A. Sigsbee, G. L. Bona, M. Taborelli, and
H. C. Siegmann, Appl. Phys. Lett. 47, 533 (1985).

[7] H.-J. Kim, J.-H. Park, and E. Vescovo, Phys. Rev. B 61, $15288(2000)$.

[8] S. A. Morton, G. D. Waddill, S. Kim, I. K. Schuller, S. A. Chambers, and J. G. Tobin, Surf. Sci. 513, L451 (2002).

[9] E. Vescovo, H.-J. Kim, J. M. Ablett, and S. A. Chambers, J. Appl. Phys. 98, 084507 (2005).

[10] M. Fonin, Y. S. Dedkov, R. Pentcheva, U. Rüdiger, and G. Güntherodt, J. Phys.: Condens. Matter 19, 315217 
(2007).

[11] M. Fonin, R. Pentcheva, Y. S. Dedkov, M. Sperlich, D. V. Vyalikh, M. Scheffler, U. Rüdiger, and G. Güntherodt, Phys. Rev. B 72, 104436 (2005).

[12] M. Fonin, Y. S. Dedkov, R. Pentcheva, U. Rüdiger, and G. Güntherodt, J. Phys.: Condens. Matter 20, 142201 (2008).

[13] D. J. Huang, C. F. Chang, J. Chen, L. H. Tjeng, A. D. Rata, W. P. Wu, S. C. Chung, H. J. Lin, T. Hibma, and C. T. Chen, J. Magn. Magn. Mater. 239, 261 (2002).

[14] S. F. Alvarado, M. Erbudak, and P. Munz, Phys. Rev. B 14, 2740 (1976).

[15] J. G. Tobin, S. A. Morton, S. W. Yu, G. D. Waddill, I. K. Schuller, and S. A. Chambers, J. Phys.: Condens. Matter 19, 315218 (2007).

[16] W. Wang, J.-M. Mariot, M. C. Richter, O. Heckmann, W. Ndiaye, P. De Padova, A. Taleb-Ibrahimi, P. Le Fèvre, F. Bertran, F. Bondino, E. Magnano, J. Krempaský, P. Blaha, C. Cacho, F. Parmigiani, and K. Hricovini, Phys. Rev. B 87, 085118 (2013).

[17] Y. S. Dedkov, U. Rüdiger, and G. Güntherodt, Phys. Rev. B 65, 064417 (2002).

[18] T. Kiss, T. Shimojima, F. Kanetaka, K. Kanaia, T. Yokoya, S. Shin, Y. Onuki, T. Togashi, C. Zhang, C. Chen, and S. Watanabe, J. Electr. Spectrosc. Relat. Phenom. 144-147, 953 (2005).

[19] S. Suga and A. Sekiyama, Photoelectron Spectroscopy: Bulk and Surface Electronic Structure (Springer, 2014).

[20] P. W. Tasker, J. Phys. C: Solid State Phys. 12, 4977 (1979).

[21] C. Noguera, J. Phys.: Condens. Matter 12, R367 (2000).

[22] T. Pohlmann, T. Kuschel, J. Rodewald, J. Thien, K. Ruwisch, F. Bertram, E. Weschke, P. Shafer, J. Wollschläger, and K. Küpper, Phys. Rev. B 102 , 220411(R) (2020).

[23] R. Pentcheva, F. Wendler, H. L. Meyerheim, W. Moritz, N. Jedrecy, and M. Scheffler, Phys. Rev. Lett. 94, 126101 (2005).

[24] L. Zhu, K. L. Yao, and Z. L. Liu, Phys. Rev. B 74, 035409 (2006).

[25] M. Paul, M. Sing, R. Claessen, D. Schrupp, and V. A. M. Brabers, Phys. Rev. B 76, 075412 (2007).

[26] A. Yanase and K. Siratori, J. Phys. Soc. Jpn. 53, 312 (1984).

[27] R. A. de Groot and K. H. J. Buschow, J. Magn. Magn. Mater. 54-57, 1377 (1986).

[28] Z. Zhang and S. Satpathy, Phys. Rev. B 44, 13319 (1991).

[29] M. Pénicaud, B. Siberchicot, C. B. Sommers, and J. Kübler, J. Magn. Magn. Mater. 103, 212 (1992).

[30] V. I. Anisimov, I. S. Elfimov, N. Hamada, and K. Terakura, Phys. Rev. B 54, 4387 (1996).

[31] A. Yanase and N. Hamada, J. Phys. Soc. Jpn. 68, 1607 (1999).

[32] V. N. Antonov, B. N. Harmon, V. P. Antropov, A. Y. Perlov, and A. N. Yaresko, Phys. Rev. B 64, 134410 (2001).

[33] V. N. Antonov, B. N. Harmon, and A. N. Yaresko, Phys. Rev. B 67, 024417 (2003).

[34] H.-T. Jeng and G. Y. Guo, Phys. Rev. B 65, 094429 (2002).

[35] H.-T. Jeng, G. Y. Guo, and D. J. Huang, Phys. Rev. Lett. 93, 156403 (2004).

[36] H.-T. Jeng, G. Y. Guo, and D. J. Huang, Phys. Rev. B 74, 195115 (2006).
[37] Z. Szotek, W. M. Temmerman, A. Svane, L. Petit, G. M. Stocks, and H. Winter, Phys. Rev. B 68, 054415 (2003).

[38] I. Leonov, A. N. Yaresko, V. N. Antonov, M. A. Korotin, and V. I. Anisimov, Phys. Rev. Lett. 93, 146404 (2004).

[39] G. K. H. Madsen and P. Novák, Europhys. Lett. 69, 777 (2005).

[40] H. P. Pinto and S. D. Elliott, J. Phys.: Condens. Matter 18, 10427 (2006).

[41] Z. Lodziana, Phys. Rev. Lett. 99, 206402 (2007).

[42] P. Piekarz, K. Parlinski, and A. M. Oleś, Phys. Rev. B 76, 165124 (2007)

[43] P. Piekarz, A. M. Oleś, and K. Parlinski, Acta Phys. Pol. A 118, 307 (2010).

[44] A. D. Rowan, C. H. Patterson, and L. V. Gasparov, Phys. Rev. B 79, 205103 (2009).

[45] X. Yu, C.-F. Huo, Y.-W. Li, J. Wang, and H. Jiao, Surf. Sci. 606, 872 (2012).

[46] R. Arras, B. Warot-Fonrose, and L. Calmels, J. Phys.: Condens. Matter 25, 256002 (2013).

[47] J. Noh, O. I. Osman, S. G. Aziz, P. Winget, and J.-L. Brédas, Sci. Technol. Adv. Mater. 15, 044202 (2014).

[48] D. Schmitz, C. Schmitz-Antoniak, A. Warland, M. Darbandi, S. Haldar, S. Bhandary, O. Eriksson, B. Sanyal, and H. Wende, Sci. Rep. 4, 5760 (2014).

[49] H. Liu and C. Di Valentin, J. Phys. Chem. C 121, 25736 (2017).

[50] H. Liu, G. Seifert, and C. Di Valentin, J. Chem. Phys. 150, 094703 (2019).

[51] O. Mounkachi, R. Lamouri, M. Hamedoun, H. EzZahraouy, E. Salmani, and A. Benyoussef, J. Supercond. Nov. Magn. 30, 3221 (2017).

[52] J. Chen, H.-S. Hsu, Y.-H. Huang, and D.-J. Huang, Phys. Rev. B 98, 085141 (2018).

[53] G. Sai Gautam and E. A. Carter, Phys. Rev. Mater. 2, 095401 (2018)

[54] S. F. Alvarado and P. S. Bagus, Phys. Lett. 67, 397 (1978).

[55] D. Schrupp, M. Sing, M. Tsunekawa, H. Fujiwara, S. Kasai, A. Sekiyama, S. Suga, T. Muro, V. A. M. Brabers, and R. Claessen, Europhys. Lett. 70, 789 (2005).

[56] X. Kozina, E. Ikenaga, C. E. Viol Barbosa, S. Ouardi, J. Karel, M. Yamamoto, K. Kobayashi, H. J. Elmers, G. Schönhense, and C. Felser, J. Electron Spectrosc. Relat. Phenom. 211, 12 (2016).

[57] O. E. Tereshchenko, V. A. Golyashov, V. S. Rusetsky, A. V. Mironov, A. Y. Demin, and A. V. V, J. Synchr. Rad. 28, 864 (2021).

[58] S. Ueda and Y. Sakuraba, Sci. Technol. Adv. Mater. 22, 317 (2021).

[59] C. Kalha, N. K. Fernando, P. Bhatt, F. O. L. Johansson, A. Lindblad, H. Rensmo, L. Z. Medina, R. Lindblad, S. Siol, L. P. H. Jeurgens, C. Cancellieri, K. Rossnagel, K. Medjanik, G. Schönhense, M. Simon, A. X. Gray, S. Nemšák, P. Lömker, C. Schlueter, and A. Regoutz, J. Phys. Condens. Matter 33, 233001 (2021).

[60] M. Paul, D. Kufer, A. Müller, S. Brück, E. Goering, M. Kamp, J. Verbeeck, H. Tian, G. Van Tendeloo, N. J. C. Ingle, M. Sing, and R. Claessen, Appl. Phys. Lett. 98, 012512 (2011).

[61] A. Müller, A. Ruff, M. Paul, A. Wetscherek, G. Berner, U. Bauer, C. Praetorius, K. Fauth, M. Przybylski, M. Gorgoi, M. Sing, and R. Claessen, Thin Solid Films 520, 368 (2011).

[62] O. Kirilmaz, Thin film growth and characterization of the 
transition metal oxides magnetite and layered perovskite iridates, Doctoral thesis, University of Würzburg (2019).

[63] A. Barbieri, W. Weiss, M. A. van Hove, and G. A. Somorjai, Surf. Sci. 302, 259 (1994).

[64] O. Fedchenko, A. Winkelmann, K. Medjanik, S. Babenkov, D. Vasilyev, S. Chernov, C. Schlueter, A. Gloskovskii, Y. Matveyev, W. Drube, B. Schönhense, H.-J. Elmers, and G. Schönhense, New J. Phys. 21, 113031 (2019).

[65] A. Winkelmann, B. Schröter, and W. Richter, Phys. Rev. B , 245417 (2004).

[66] C. Schlueter, A. Gloskovskii, K. Ederer, I. Schostak, S. Piec, I. Sarkar, Y. Matveyev, P. Lömker, M. Sing, R. Claessen, C. Wiemann, C. M. Schneider, K. Medjanik, G. Schönhense, P. Amann, A. Nilsson, and W. Drube, in 13th International Conference on Synchrotron Radiation Instrumentation - SRI2018, Vol. 2054 (AIP Conference Proceedings, Taipei, Taiwan, 2019) p. 040010.

[67] Note that the sample could not be fully magnetized $e x$ situ and had to be measured in remanence due to technical limitations. Hence, the XMCD signal is weaker compared to previously published absorption spectra. Nevertheless, the lineshape of the XMCD signal is in very good agreement with those data.

[68] P. Kuiper, B. Searle, L.-C. Duda, R. Wolf, and P. van der Zaag, J. Electron Spectrosc. 86, 107 (1997).

[69] J. Chen, D. J. Huang, A. Tanaka, C. F. Chang, S. C. Chung, W. B. Wu, and C. T. Chen, Phys. Rev. B 69, 085107 (2004)

[70] E. Goering, Phys. Status Solidi B 248, 2345 (2011).

[71] E. Goering, S. Gold, M. Lafkioti, and G. Schütz, Europhys. Lett. 73, 97 (2006).

[72] J. Schlappa, C. Schüßler-Langeheine, C. F. Chang, H. Ott, A. Tanaka, Z. Hu, M. W. Haverkort, E. Schierle, E. Weschke, G. Kaindl, and L. H. Tjeng, Phys. Rev. Lett. 100, 026406 (2008).

[73] D. Yu, C. Math, M. Meier, M. Escher, G. Rangelov, and M. Donath, Surf. Sci. 601, 5803 (2007).

[74] Y. S. Dedkov, M. Fonin, D. V. Vyalikh, J. O. Hauch, S. L. Molodtsov, U. Rüdiger, and G. Güntherodt, Phys. Rev. B 70, 073405 (2004).

[75] We chose $5 \mathrm{keV}$ as a good compromise between the dropping cross section at higher photon energies and the increase of third-order radiation from the undulator at lower energies.

[76] M. Paul, Molecular Beam Epitaxy and Properties of Magnetite Thin Films on Semiconducting Substrates, Doc- toral thesis, University of Würzburg (2010).

[77] D. Kutnyakhov, P. Lushchyk, A. Fognini, D. Perriard, M. Kolbe, K. Medjanik, E. Fedchenko, S. A. Nepijko, H.-J. Elmers, G. Salvatella, C. Stieger, R. Gort, T. Bähler, T. Michlmayer, Y. Acremann, A. Vaterlaus, F. Giebels, H. Gollisch, R. Feder, C. Tusche, A. Krasyuk, J. Kirschner, and G. Schönhense, Ultramicroscopy 130, 63 (2013).

[78] P. G. Steeneken, L. H. Tjeng, I. Elfimov, G. A. Sawatzky, G. Ghiringhelli, N. B. Brookes, and D.-J. Huang, Phys. Rev. Lett. 88, 047201 (2002).

[79] L. L. Lev, D. V. Averyanov, A. M. Tokmachev, F. Bisti, V. A. Rogalev, V. N. Strocov, and V. G. Storchak, J. Mater. Chem. C 5, 192 (2017).

[80] J. P. Wright, J. P. Attfield, and P. G. Radaelli, Phys. Rev. Lett. 87, 266401 (2001).

[81] J. P. Wright, J. P. Attfield, and P. G. Radaelli, Phys. Rev. B 66, 214422 (2002).

[82] S. F. Alvarado, Z. Phys. B 33, 51 (1979).

[83] D. Mauri, D. Scholl, H. C. Siegmann, and E. Kay, Phys. Rev. Lett. 61, 758 (1988).

[84] Note that recently a total energy resolution (photon band width plus analyzer resolution) as good as $60 \mathrm{meV}$ has been demonstrated for spin-integrated HAXPES operation of the same momentum microscope used here [87]. Although the additional use of the spin-filter crystal results in a significant count rate reduction, this result represents an encouraging prospect for future spin-HAXPES experiments.

[85] G. Ghiringhelli, K. Larsson, and N. B. Brookes, Rev. Sci. Instrum. 70, 4225 (1999).

[86] M. Kolbe, P. Lushchyk, B. Petereit, H.-J. Elmers, G. Schönhense, A. Oelsner, C. Tusche, and J. Kirschner, Phys. Rev. Lett. 107, 207601 (2011).

[87] K. Medjanik, S. V. Babenkov, S. Chernov, D. Vasilyev, B. Schönhense, C. Schlueter, A. Gloskovskii, Y. Matveyev, W. Drube, H.-J. Elmers, and G. Schönhense, J. Synchrotron Radiat. 26, 1996 (2019).

[88] G. Schönhense, K. Medjanik, S. Chernov, D. Kutnyakhov, O. Fedchenko, M. Ellguth, D. Vasilyev, A. Zaporozhchenko-Zymaková, D. Panzer, A. Oelsner, C. Tusche, B. Schönhense, J. Braun, J. Minár, H. Ebert, J. Viefhaus, W. Wurth, and H.-J. Elmers, Ultramicroscopy 183, 19 (2017).

[89] S. Suga and C. Tusche, J. Electr. Spectrosc. Relat. Phenom. 200, 119 (2015). 